\title{
Filtragem Online Baseada na Informação do Sistema Calorimetria do Experimento ATLAS Utilizando Modelos de Aprendizado de Máquinas
}

\author{
Micael Veríssimo de Araújo*, \\ João Victor da Fonseca Pinto, \\ José Manoel de Seixas \\ Laboratório de Processamento de Sinais COPPE/UFRJ \\ Universidade Federal do Rio de Janeiro \\ Rio de Janeiro, Brasil \\ e-mail: *micael.verissimo@lps.ufrj.br
}

\begin{abstract}
Na área de Física Experimental de Altas Energias uma etapa sensível é a aquisição dos dados com os quais os físicos, a partir de suas análises, colocam à prova as mais diversas teorias. Assim, os experimentos construídos possuem, dentre muitas outras características, uma grande quantidade de canais de leitura e interesse por eventos raros. Neste contexto destacase o sistema de filtragem (Trigger) empregado pelo ATLAS tem utilizado desde 2017 uma estratégia de seleção baseada em um conjunto de redes neurais, chamado NeuralRinger apenas para candidatos com energia transversa superior a $15 \mathrm{GeV}$. $O$ NeuralRinger faz uso das características naturais de deposição de energia dos elétrons no sistema de calorimetria de modo a encapsular estas características em anéis, que por sua vez são utilizados como entradas para as redes neurais que tomam a decisão de filtragem. O ATLAS está próximo de voltar a coletar dados e para esta nova etapa o NeuralRinger expandirá sua atuação para à toda região cinemática. Neste trabalho serão exibidos resultados simulados obtidos pelo NeuralRinger para a região abaixo de $15 \mathrm{GeV}$.
\end{abstract}

Index Terms-Redes Neurais, Processamento Online, Calorimetria

\section{INTRODUÇÃO}

Nas mais diversas áreas das engenharias não é difícil encontrar ambientes com alta dimensionalidade envolvida, onde buscam-se por sinais raros inseridos em elevadas taxas de amostragem sob uma grande quantidade de ruído que falseiam o sinal de interesse. Estas características estão presentes em aplicações que tangem áreas como telecomunicações, óleo e gás, controle, eletricidade, etc. Posto isto, nos diversos casos as engenharias tem se beneficiado do uso de técnicas de inteligência computacional.

A física de altas energias, assim como nas engenharias, é uma área onde podemos encontrar as características que foram descritas acima. Esta área de conhecimento busca, compreender os constituintes básicos da matéria que compõem o universo bem como as relações entre eles [1].

Neste contexto, destaca-se o experimento ATLAS (sigla para $\boldsymbol{A}$ Toroidal LHC ApparatuS) [2] um dos quatro grande experimentos presentes no LHC (Large Hadron Collider) [3] (veja Fig. 1), o maior colisionador de partículas do mundo com $27 \mathrm{~km}$ de comprimento de circunferência. As colisões produzidas pelo LHC e as análises produzidas pelos experimentos que as coletam, sendo um destes o ATLAS, foram essenciais para que em 2012 os físicos P.W. Higgs e F. Englert fossem laureados com o prêmio nobel de física do ano de 2012 [4].

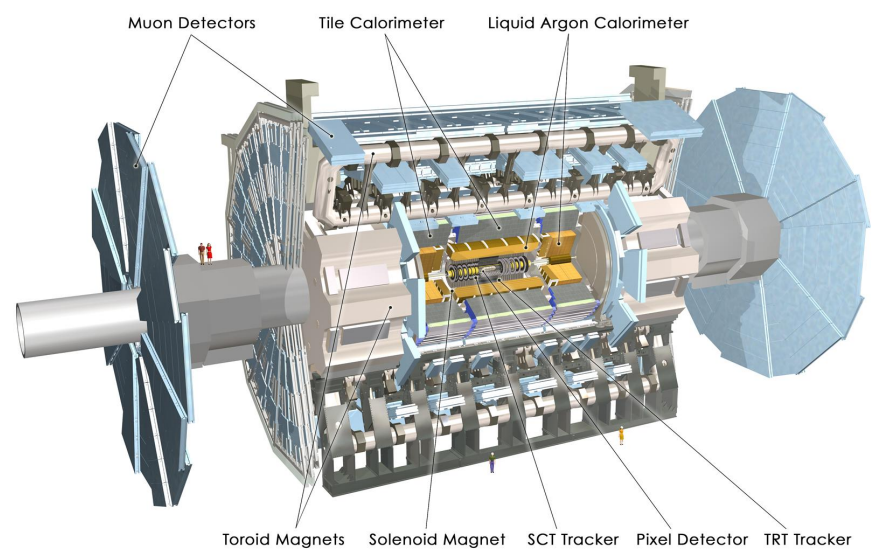

Fig. 1. O detector ATLAS e seus subsistemas. Fonte: [5].

O ATLAS é o maior experimento do LHC, possuindo 44 metros de comprimento e 25 metros de altura. O ATLAS é um experimento de dito de propósito geral, ou seja, seu objetivo é estudar todas os processos físicos gerados nas colisões do LHC. O ATLAS tem um formato cilíndrico e se subdivide em 3 sistemas principais: o Detetor de Traços (ID) responsável por medir a trajetória das partículas carregadas; os calorímetros ${ }^{1}$, que realizam a medição da energia das partículas absorvendo-as [6]; e por fim, a câmara de múons, especificamente desenhada para detectar os múons, partículas

${ }^{1} \mathrm{O}$ sistema de calorimetria do ATLAS possui ao todo 7 camadas de amostragem, sendo uma de pré-amostragem, três camadas eletromagnéticas e três camadas hadrônicas 
que assim como elétrons são importantes e estão presentes em grande parte dos processos físicos que o ATLAS busca estudar.

O sistema de calorimetria do ATLAS é formado por dois tipos de calorímetros [7]: o calorímetro eletromagnético (ECAL) e o calorímetro hadrônico (HCAL).

O calorímetro eletromagnético realiza a medição da energia das partículas que interagem por meio da força eletromagnética; enquanto por sua vez o calorímetro hadrônico tem a capacidade de medir a energia das partículas que interagem por meio da força forte.

No LHC, a cada 25 ns uma colisão acontece, gerando aproximadamente 1,7 MB por evento para o ATLAS. Devido a sua estrutura altamente segmentada seriam necessários adquirir em torno de 70 TB/s [8], o que é impossível de ser realizável e por isso a necessidade de um sistema de filtragem online (Trigger) para as assinaturas de interesse.

O sistema de Trigger do ATLAS faz uso de reconhecimento de padrões para realizar a identificação dos objetos físicos de interesse. A filtragem é feita em dois níveis [9]. O primeiro nível (L1), totalmente implementado em hardware, tem o objetivo de reduzir a taxa de entrada para $100 \mathrm{kHz}$. O segundo nível é totalmente implementado em software chamado de High Level Trigger (HLT), tem por objetivo manter a taxa média de saída em $1,5 \mathrm{kHz}$ mantendo uma latência média em torno de $200 \mathrm{~ms}$. O HLT é subdividido em duas etapas, a saber, rápida (fast) e precisa (precision). Durante a etapa rápida são utilizadas as informações dos sistemas que possuem menor latência, como é o caso o sistema de calorimetria, com posterior adição de parte da informação do Detector de Traços; enquanto na etapa precisa, são utilizados algoritmos de maior latência, como por exemplo, os algoritmos de processamento de imagem utilizados na identificação da trajetória das partículas carregadas.

Neste trabalho o foco está nos canais (ou cadeias que aplicam um ou mais critérios de seleção) de elétrons. Os elétrons são partículas importantes, visto que estão presentes em muitos processos físicos de interesse do ATLAS, como por exemplo o decaimento do bóson de Higgs que em alguns casos envolvem até 2 elétrons (e 2 anti-elétrons, conhecido como pósitrons). No ATLAS a identificação dos elétrons é feita principalmente com o uso da informação do sistema de calorimetria e do ID. Visto que no ID operam algoritmos que fazem uso de processamento de imagem, a filtragem apenas utilizando a informação de calorimetria é uma forma de reduzir a latência e otimizar os recursos disponíveis durante a tomada de dados.

A quantidade de colisões produzidas pelo LHC vem aumentando graças ao processo de colimação dos feixes de prótons, isto implica em maior quantidade de dados a serem coletados. Entretanto, este processo também gera um fenômeno conhecido pile up [10], onde os sinais medidos pelo detector sofrem o empilhamento devido ao alto número de colisões que ocorrem. Assim, devido a este efeito a tomada de decisão do torna-se mais difícil, devido as distorções causadas pela maior ocupação do detector.
Para conter os efeitos do pile up, o ATLAS, em 2017, alterou a estratégia utilizada na etapa rápida do HLT na filtragem de elétrons, passando a fazer uso de um conjunto de redes neurais (NeuralRinger) para tomada a decisão. O NeuralRinger [8], [11] faz uso apenas da informação oriundo do sistema de calorimetria, sendo esta compactada no sob a forma de anéis concêntricos de deposição de energia. Inicialmente o NeuralRinger foi utilizando apenas na filtragem de elétrons com energia transversa ${ }^{2}\left(E_{T}\right)$ maior ou igual à 15 $\mathrm{GeV}$, enquanto abaixo desta faixa, operou um conjunto de cortes sequenciais em variáveis extremamente discriminantes. As variáveis e a sequência de cortes são: : $R_{\eta}, E_{\text {ratio }}, E_{T, E M}$ e $R_{\text {had }}$.

- $R_{\eta}$ (vazamento em $\eta$ ): definida na segunda camada eletromagnética (EM2) como:

$$
R_{\eta}=\frac{E_{3 \times 7}}{E_{7 \times 7}},
$$

onde $E_{m \times n}$ é a energia depositada em uma região de tamanho $m \times n$ células em $\eta \times \phi$ ao redor da célula mais quente da EM2 (com maior energia depositada).

- $E_{\text {ratio }}$ : definida como a razão da diferença de energia entre o maior e o segundo maior depósito de energia e a soma dessas energias na primeira camada eletromagnética (EM1).

$$
E_{\text {ratio }}=\frac{E_{\max , 1}^{S 1}-E_{\max , 2}^{S 1}}{E_{\max , 1}^{S 1}+E_{\max , 2}^{S 1}}
$$

- $E_{T, E M}$ (Energia Transversa Eletromagnética): definida como a energia transversa total que foi depositada ao longo das três camadas do calorímetro eletromagnético (EM1, EM2, EM3), em uma região de tamanho $3 \times 7$ células, centrada na célula mais quente da EM2.

- $R_{\text {had }}$ (vazamento hadrônico): definida como:

$$
R_{h a d}=\frac{E_{T}^{h a d}}{E_{T}^{E M}},
$$

onde $E_{T}^{\text {had }}$ é a energia transversa medida no HCAL e $E_{T}^{E M}$ é a energia transversa medida no ECAL.

Este artigo, apresentará a extensão do algoritmo NeuralRinger para a região com $E_{T}<15 \mathrm{GeV}$. Nesta região, existe maiores flutuações e a resolução do detector é deteriorada, visto que a mesma é proporcional à $1 / \sqrt{E}$ [6]. $\mathrm{O}$ artigo possui a seguinte organização: na Seção II encontra-se a descrição, do algoritmo NeuralRinger bem como o método utilizado para sua extensão. A discussão dos resultados obtidos será exposta na Seção III seguida das conclusões na Seção IV.

\section{NeuralRinger}

Durante as colisões os elétrons terão sua energia absorvida nos calorímetros gerando um chuveiro de partículas [6]. Este chuveiros por sua vez são descritos por meio de grandezas físicas que levam em consideração largura lateral e longitudinal do chuveiro que fora medido nos calorímetros. Esta

\footnotetext{
${ }^{2} \mathrm{~A}$ energia transversa de um objeto com massa $m$ e momento tansverso $p_{T}$ é dada por: $E_{T}=\sqrt{m^{2}+p_{T}^{2}}$.
} 
informação é de extrema importância quando se está interessado em caracterizar os chuveiros formados por elétrons. Sobre os chuveiros eletromagnéticos (formados por elétrons ou fótons) sabe-se que em torno de $90 \approx 99 \%$ de sua energia fica contida em um cilíndro com raio entre $1 \mathrm{e}$ 3,5 $R_{M}$, onde $R_{M}$ é o raio de Molière ${ }^{3}$ e se alastram por uma região lateralmente mais ampla do calorímetro, conforme se desenvolve longitudinalmente obtendo assim um aspecto cônico.

Uma forma diferente de obter estas características do chuveiro é através da construção de anéis concêntricos de deposição energia [13]. O NeuralRinger utiliza esta formatação por anéis, e para cada anel, temos a soma da energia depositadas nas células que compõem uma Região de Interesse (Region of Interest - RoI), selecionada no primeiro nível de filtragem. Este processo é repetido ao longo de todas as camadas de amostragem do sistema de calorimetria do ATLAS permitindo assim, a compactação das informações de largura longitudinal dos chuveiros (conforme ilustração da Fig. 2). São construídos 100 anéis ao longo de todo sistema de calorimetria do ATLAS, conforme visto na Tabela I.
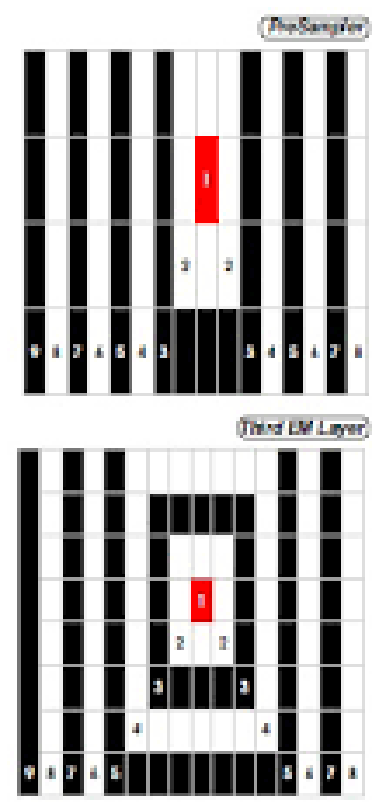

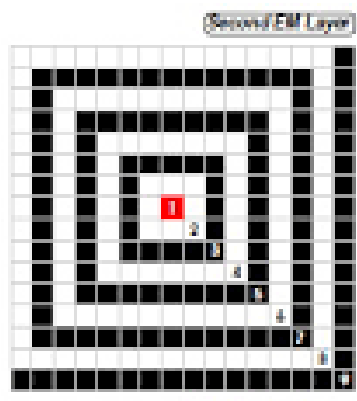

frat Hindronk Love

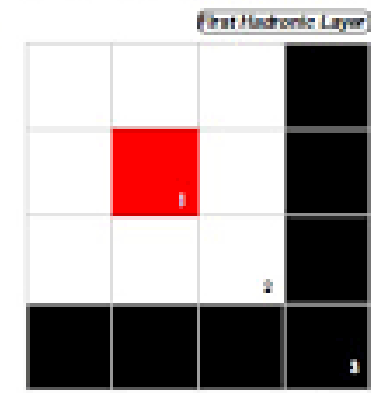

Fig. 2. Ilustração da reconstrução dos anéis ao longo das camadas do calorímetro eletromagnético e a primeira camada do calorímetro hadrônico. Fonte: [11].

Os processos físicos gerados pelas iterações dos elétrons com os materiais que compõem os calorímetros sofrem alterações em função da energia; de forma semelhante, visando manter a informação mais uniforme possível ao longo do sistema de calorimetria, o Trigger adotou uma segmentação da decisão por regiões específicas de $E_{T}$ e $\eta$ (posição no detector). Com isto, o NeuralRinger tem atuado na etapa

\footnotetext{
${ }^{3}$ Por definição, o raio de Molière é o raio do cilindro com eixo coincidindo com o eixo do chuveiro. Este contém, em média, $90 \%$ da deposição de energia do chuveiro [12].
}

Tabela I

NúMERO DE ANÉIS POR CAMADA DOS CALORÍMETROS. SENDO PS O CAlorímetro Pré-AMOSTRAdor; EM1,2,3 REFERENTES AS CAMADAS Do CALORÍMETro Eletromagnético; E HAD1,2,3 REFERENTE AS CAMADAS DO CALORÍMETRO HADRÔNICO.

\begin{tabular}{|c|c|c|c|c|c|c|c|}
\hline PS & EM1 & EM2 & EM3 & HAD1 & HAD2 & HAD3 & Total \\
\hline 8 & 64 & 8 & 8 & 4 & 4 & 4 & 100 \\
\hline
\end{tabular}

rápida da HTL com um total de 25 redes neurais para tomar a decisão de filtragem apenas baseado nos 100 fornecidos pelo sistema de calorimetria [8], [11]. Assim, a cada novo candidato uma rede neural, selecionada de acordo com a posição e energia do candidato, assume a decisão da filtragem.

\section{A. Extensão para $E_{T}<15 \mathrm{GeV}$}

Conforme dito anteriormente o NeuralRinger tinha sua atuação limitada apenas aos canditados com $E_{T} \geq 15$ $\mathrm{GeV}$, assim uma extensão natural seria abranger a área com $E_{T}<15 \mathrm{GeV}$ já no próximo período de coleta de dados (Run 3). Neste região de menor energia, a identificação dos chuveiros é degradada devido a menor resolução de energia dos calorímetros.

O ensemble que compõem o NeuralRinger nesta região é composto por 3 faixas de $E_{T}$ que subdividem a região entre $4 \leq E_{T}<15$ e 5 faixas de $\eta$ que subdividem a região entre $0 \leq \eta<2,47$, totalizando 15 regiões conforme descrito na Tabela II.

\begin{tabular}{c}
\hline \hline Ajuste do Modelo \\
\hline \hline Fronteira das Regiões em $E_{T}[\mathrm{GeV}]$ \\
\hline $4 \leq E_{T}<7$ \\
$7 \leq E_{T}<10$ \\
$10 \leq E_{T}<15$ \\
\hline Fronteiras das Regiões em $|\eta|$ \\
$0,0 \leq|\eta|<0,8$ \\
$0,8 \leq|\eta|<1,37$ \\
$1,37 \leq|\eta|<1,54$ \\
$1,54 \leq|\eta|<2,37$ \\
$2,37 \leq|\eta|<2,47$ \\
\hline \hline
\end{tabular}

Tabela II

FRONTEIRAS PARA AS REGIÕES EM $\eta$ E $E_{T}$ EMPREGADAS NO AJUSTE DOS MODELOS PARA O NeuralRinger ABAIXO DE 15 GEV. UM TOTAL DE 15 REGIÕES SÃO UTILIZADAS PARA COMPOR O ensemble DE REDES NEURAIS.

Abaixo de $15 \mathrm{GeV}$, a maior fonte de elétrons (sinal) é advinda do decaimento do méson $J / \Psi$, onde o mesmo decaí em um par elétron-pósitron. Posto isto, o conjunto de dados utilizados para os treinamentos e testes dos modelos é obtido a partir de elétrons vindos deste decaimento. Todas as amostras foram selecionadas com base no conhecimento especialista conforme descrito em [14]. As amostras de ruído são compostas por candidatos que não foram aprovados, do ponto de vista de seleção offline, por nenhum dos critérios empregados pelo ATLAS, neste casos jatos hadrônicos. Os dados utilizados neste trabalho compreendem os períodos de colisão de 2017 e de 2018, optando-se por desenvolver os modelos utilizando-se 
dos dados de 2017 e emulando a operação do Trigger com os dados de 2018.

Cada um dos modelos que compõem o ensemble é uma rede neural do tipo Multilayer perceptron (MLP) com uma única camada escondida e um neurônio do tipo tangente hiperbólica na saída, assim como os neurônios da camada escondida. Para o treinamento optou-se por utilizar o algoritmo ADAM [15] e a função custo utilizada foi o Erro Médio Quadrático (MSE). $\mathrm{O}$ critério de para empregado nos treinamentos baseou-se na maximização do índice SP (Eq.(4)) [16]-[18].

$$
S P=\sqrt{\sqrt{P_{D} \cdot\left(1-P_{F}\right)} \cdot \frac{P_{D}+\left(1-P_{F}\right)}{2}}
$$

onde $P_{D}$ é a probabilidade de detecção de elétrons e $P_{F}$ é a probabilidade de classificar como elétron um jato hadrônico.

Para estimar a incerteza estatística associada aos modelos obtidos foi empregado o método de validação cruzada Stratified $K$-Fold com $K=10$ [19], onde o conjunto de treinamento é subdivido em 10 partes que tenham uma representação apropriada do conjunto de treino em termos de distribuição de classes, média, variância e etc. Assim 9 destas partes são utilizadas para o treinamento dos modelos e 1 é utilizada para teste do mesmo, obtendo assim uma configuração treino/teste. Este processo então é repetido alternando-se a partição que será utilizada como teste, totalizando 10 configurações de treino/teste. Também foram realizadas diferentes inicializações em cada configuração, neste caso foram feitas para cada uma das 10 configurações 5 inicializações para evitar o mínimo local. Cada inicialização possui um limite de 5000 épocas de treinamento e o tamanho da batelada utilizada foi de $1024^{4}$.

A quantidade de neurônios empregada na camada escondida foi feita através de uma busca, limitando-se a faixa entre 2 até 10 neurônios. A escolhe final se deu avaliando-se o índice SP por meio de boxplots buscando-se sempre manter a parcimônia.

O ATLAS possui diversos interesses nos mais diversos processos físicos. Por isto, alguns o Trigger possui alguns critérios, baseados na pureza da amostras, que são tomados em consideração durante a aquisição de dados e estes são:

- Tight: Neste critério, é priorizada a qualidade da amostra.

- Medium: Critério que visa o equilíbrio entre a pureza da amostra e a estatística do processo de interesse.

- Loose: Este é um critério que prioriza o volume estatístico da amostra.

- Very Loose: É o critério loose mais eficiente na rejeição da contaminação.

Neste trabalho, no conjunto de treinamento foi exigido que o sinal seja aprovado pelo critério Medium e o ruído, como exposto anteriormente, exigiu-se a reprovação em todos os critérios listados acima.

Uma vez que os modelos estejam treinandos os mesmos precisam ser ajustados de modo a operar atendendo a estes critérios e mantendo a detecção, assim as maiores

\footnotetext{
${ }^{4}$ Nos casos onde uma das classe possuir menos eventos do que 1024 adotouse como batelada a quantidade de eventos da menor classe.
}

\begin{tabular}{lcc}
\hline \hline & $P_{D}[\%]$ & $P_{F}[\%]$ \\
\hline Ref. (CutBased) & 97,78 & 36,91 \\
15 MLPs com 2 neurônios & $97,78 \pm 0,01$ & $10,30 \pm 0,30$ \\
15 MLPs com 5 neurônios & $97,79 \pm 0,01$ & $10,30 \pm 0,38$ \\
15 MLPs com 10 neurônios & $97,78 \pm 0,01$ & $10,27 \pm 0,32$ \\
Tabela III &
\end{tabular}

RESULTADOS INTEGRADOS DA VALIDAÇÃO CRUZADA CONSIDERANDO TODAS REGIÕES.

contribuições desta extensão é vista na redução da taxa de falso alarme (quantidade de ruído classificado erroneamente como elétrons).

Assim, uma vez que os modelos estejam treinados e ajustados para operar conforme as exigências do sistema de Trigger é possível realizar emulações das cadeias de filtragem de modo a avaliar a performance do NeuralRinger em comparação ao sistema baseado em cortes (CutBased).

\section{RESULTADOS}

A quantidade de neurônios na camada escondida escolhida para cada modelo do ensemble foi de 2 neurônios, uma vez que todos os modelos obtiveram performance similar, conforme ilustra a Fig. 3. Na Tabela III estão dispostos os valores da validação cruzada integrando-se todas as 15 regiões levando-se em consideração a operação Tight.

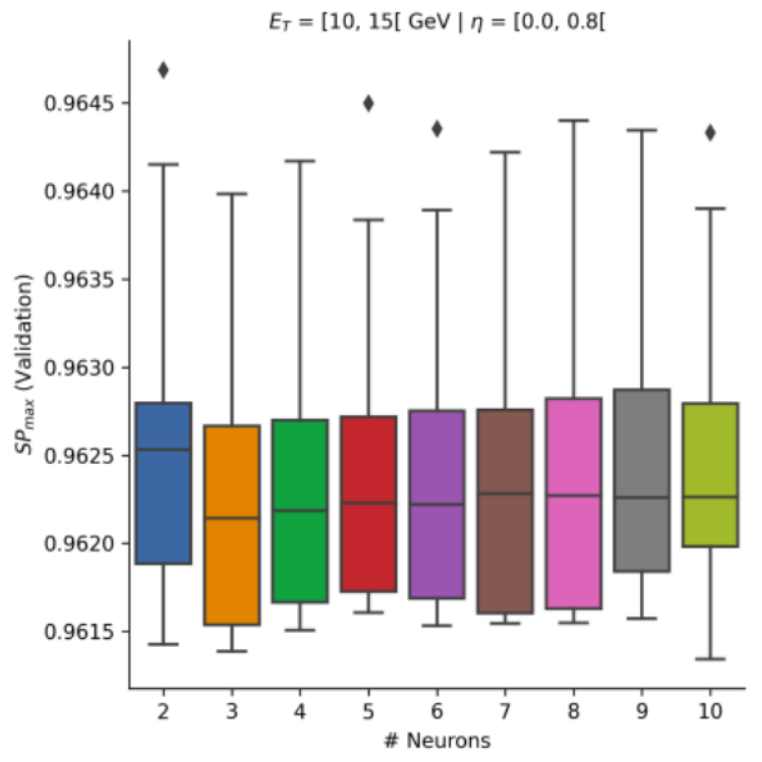

Fig. 3. Boxplot dos modelos testados para região entre $10 \mathrm{GeV} \leq E_{T}<$ $15 \mathrm{GeV}$ e $0 \leq \eta<0.8$. Este comportamento foi visto nas outras regiões do ensemble.

Nota-se que com apenas 2 neurônios é possível manter-se a detecção reduzindo-se o o falso alarme por um fator de $3 x$. Desta forma todas em regiões as foram utilizados modelos com 2 neurônios e prosseguiu-se para emulação do sistema de Trigger.

A emulação do Trigger foi feita utilizando-se dados de 2018 e avaliou-se quatro cadeias de seleção a saber:

- HLT_e5_lhtight_nod0: nesta cadeia são empregados cortes Tight aos canditados com $E_{T}>5 \mathrm{GeV}$; 
- HLT_e5_lhmedium_nod0: nesta cadeia são empregados cortes Medium aos canditados com $E_{T}>5 \mathrm{GeV}$;

- HLT_e5_lhloose_nod0: nesta cadeia são empregados cortes Loose aos canditados com $E_{T}>5 \mathrm{GeV}$;

- HLT_e5_lhvloose_nod0: nesta cadeia são empregados cortes Very Loose aos canditados com $E_{T}>5 \mathrm{GeV}$;

Cada cadeia foi duplicada, assim em uma realizou-se a emulação utilizando o algoritmo padrão CutBased e em outra utilizou-se o NeuralRinger para tomada de decisão. Para evitar viés vindo do NeuralRinger que já opera desde 2017, filtrou-se os dados para os mesmos tenha $E_{T}<15 \mathrm{GeV}$. Na Tabela IV estão dispostos os resultados obtidos pela emulação do sistema de filtragem, onde nota-se a redução do $P_{F}$ tanto durante a etapa rápida quanto ao final da cadeia de processamento por completo e na Fig. 4 podemos ver a rejeição do Trigger para a cadeia HLT_e5_lhtigh_nod0 operando com NeuralRinger e operando com o CutBased, medida em forma de eficência em ruído. Já na Fig. 5 vemos a eficiência em sinal em função de $E_{T}$ do candidato e verifica-se que ambos algoritmos operam de forma similar obtendo valores próximos de eficiência.

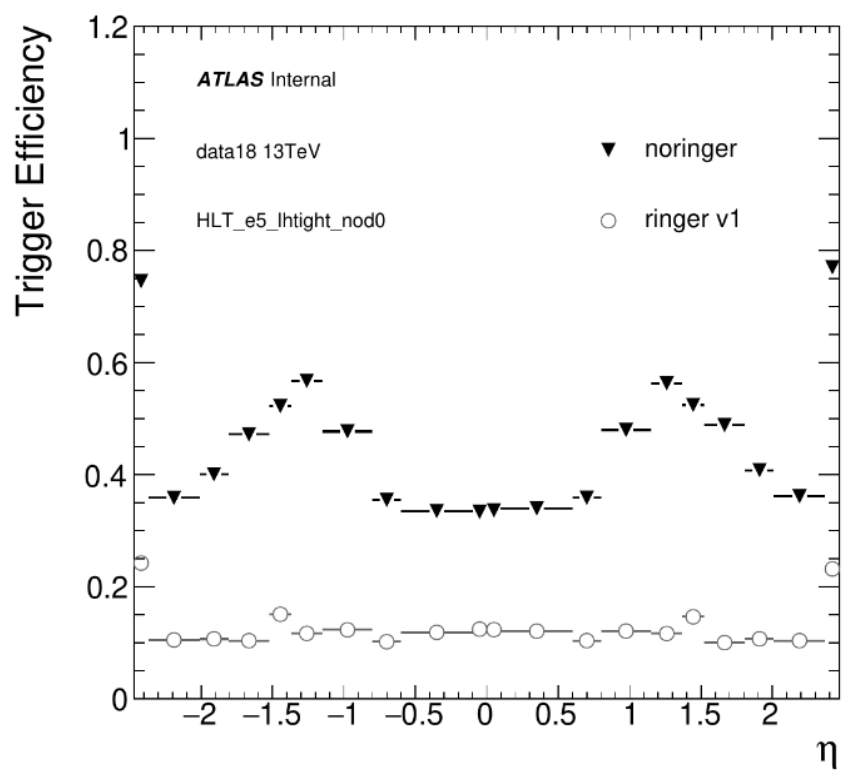

Fig. 4. Eficiência na decteção de ruído para a cadeia operando com o NeuralRinger (marcador aberto) e CutBased (marcador fechado).

Para avaliar de forma quantitativa o desempenho do NeuralRinger realizou-se uma análise de quadrante. Esta análise consiste em dividir o espaço de decisão em quatro regiões mutuamente exclusivas caracterizando quatro casos diferentes que podem ocorrer durante a emulação da cadeia HLT_e5_lhtight_nod0 estes casos são:

- Caso 1: a cadeia com NeuralRinger e a cadeia com CutBased aceitam o canditado;

- Caso 2: apenas a cadeia com CutBased aceita o candidato;

- Caso 3: apenas a cadeia com NeuralRinger aceita o candidato;

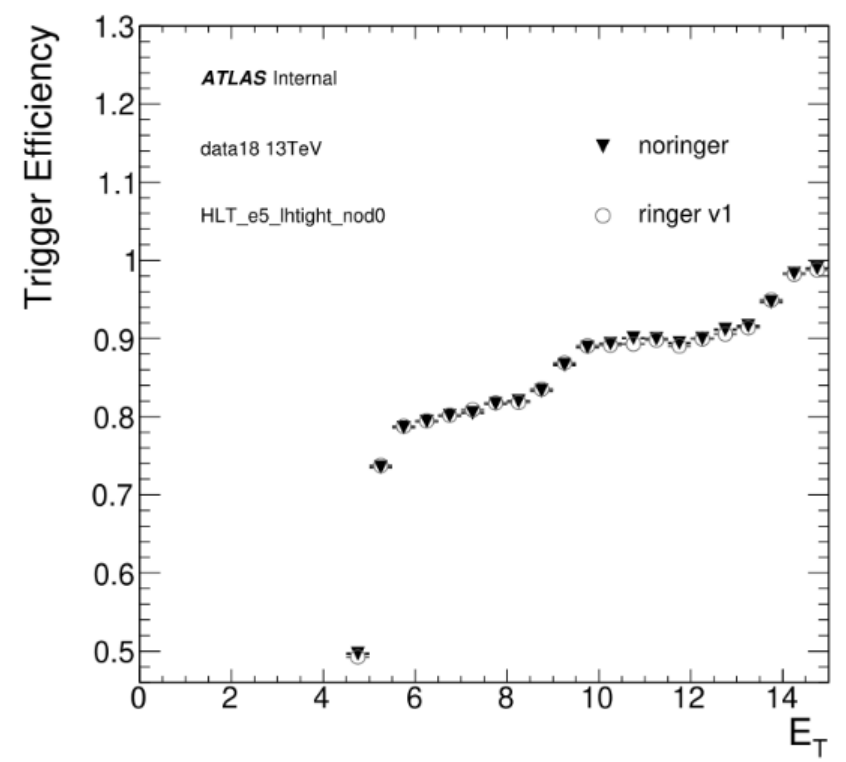

Fig. 5. Deteção de eventos de sinal para a cadeia operando com o NeuralRinger (marcador aberto) e CutBased (marcador fechado).

- Caso 4: a cadeia com NeuralRinger e a cadeia com CutBased rejeitam o canditado.

A partir das variáveis utilizadas na identificação de elétrons offline, uma vez que a identificação offline é balizador para o Trigger, verificou-se a ocorrência destes casos. Pelo conhecimento especialista da física é esperado, por exemplo, que elétrons aprovados por um critério Tight exibam valores da variável $E_{\text {ratio }}$ o mais próximo de 1 possível. Na Fig. 6 estão expostos os resultados da análise de quadrante.

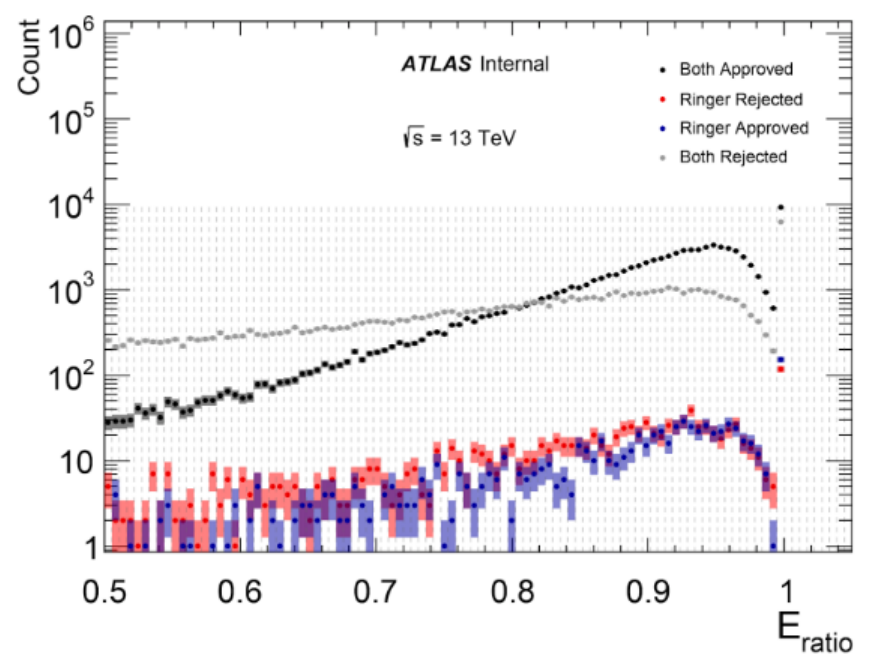

Fig. 6. Análise de quadrante para variável $E_{\text {ratio }}$ para a emulação da cadeia HLT_e5_lhtight_nod0. Em preto encontra-se o Caso 1; em vermelho o Caso 2; em azul o Caso 3 e em cinza o Caso4.

Comparando as respostas, observou-se que o NeuralRinger obteve valores condizentes com o esperado para a variável $E_{\text {ratio }}$ e que na maioria dos casos avaliados ambas as cadeias 


\begin{tabular}{ccc|c|cc|c}
\hline \hline Cadeias & \multicolumn{3}{c}{ NeuralRinger } & \multicolumn{3}{c}{ CutBased } \\
& $P_{D}[\%]$ & $P_{F}[\%]$ & $P_{F_{\text {fast }}}[\%]$ & $P_{D}[\%]$ & $P_{F}[\%]$ & $P_{F_{f a s t}}[\%]$ \\
\hline HLT_e5_lhtight_nod0 & 87,35 & 0,15 & 11,68 & 87,33 & 0,16 & 43,00 \\
HLT_e5_lhmedium_nod0 & 85,40 & 0,22 & 12,92 & 85,41 & 0,25 & 46,50 \\
HLT_e5_lhloose_nod0 & 90,71 & 1,14 & 14,97 & 90,93 & 1,41 & 48,21 \\
HLT_e5_lhvloose_nod0 & 93,45 & 4,18 & 14,97 & 94,72 & 6,35 & 53,19
\end{tabular}

RESUltados da EMUlaÇÃo do Sistema de Trigger aO FINAL DAS CADEIAS DE PROCESSAMENTO E O FALSO ALARME OBTIDO DURANTE A ETAPA RÁPIDA DE FILTRAGEM.

concordaram, seja em rejeitar o candidato, seja para aceitar o candidato.

\section{Conclusões}

Os experimentos de físicas de altas energias proporcionam para o campo de aprendizado de máquinas um ambiente propício ao desenvolvimento e avaliação de modelos, devido a grande quantidade de dados gerados por seus experimentos, a alta dimensionalidade advinda da fina segmentação dos detectores e a enorme quantidade de ruído que falseia o sinal de interesse.

A extensão do NeuralRinger para a região de $E_{T}<15 \mathrm{GeV}$ irá proporcionar ao ATLAS melhor desempenho durante a etapa rápida do Trigger, visto que os resultados de emulação apontam para uma drástica redução do ruído durante o processamento da cadeia, principalmente durante a etapa rápida, mesmo mantendo-se a mesma taxa de detecção.

A análise de quadrante também sugere um bom acordo entre entre o NeuralRinger e as variáveis utilizadas para a identificação offline de elétrons nesta região.

Desta forma os resultados deste trabalho apontam que a extensão do NeuralRinger obteve resultados satisfatórios, superando a atual seleção baseada em cortes.

\section{AGRADECIMENTOS}

Os autores agradecem ao CNPq, FAPEMIG, FAPESB, FAPERJ e RENAFAE pelo apoio financeiro. O presente trabalho foi realizado com apoio da Coordenação de Aperfeiçoamento de Pessoal de Nível Superior - Brasil (CAPES) - Código de Financiamento 001.

\section{REFERÊNCIAS}

[1] Griffiths, David. "Introduction to elementary particles." John Wiley \& Sons, 2020.

[2] Aad, Georges, et al. "The ATLAS experiment at the CERN large hadron collider." Journal of instrumentation 3 (2008): S08003.

[3] Evans, Lyndon, and Philip Bryant. "LHC machine." Journal of instrumentation 3.08 (2008): S08001.

[4] The Nobel Prize in Physics 2013. NobelPrize.org. Nobel Media AB 2019. Fri. 24 May 2019. https://www.nobelprize.org/prizes/physics/2013/summary/.

[5] Pequenao, Joao. Computer generated image of the whole ATLAS detector. No. CERN-GE-0803012. 2008.

[6] Wigmans, Richard, and Richard Wigmans. Calorimetry: Energy measurement in particle physics. Vol. 107. Oxford University Press, 2000.

[7] Puzo, P. "ATLAS calorimetry." Nuclear Instruments and Methods in Physics Research Section A: Accelerators, Spectrometers, Detectors and Associated Equipment 494.1-3 (2002): 340-345.

[8] da Fonseca Pinto, Joao Victor. "Ring-shaped Calorimetry Information for a Neural Egamma Identification with ATLAS Detector." Journal of Physics: Conference Series. Vol. 762. No. 1. IOP Publishing, 2016.
[9] Ruiz Martínez, Aranzazu, and ATLAS Collaboration. "The Run-2 ATLAS Trigger System." Journal of Physics Conference Series. Vol. 762. No. 1. 2016.

[10] Marshall, Zachary. "Simulation of Pile-up in the ATLAS Experiment." Journal of Physics: Conference Series. Vol. 513. No. 2. IOP Publishing, 2014.

[11] Freund, Werner Spolidoro. "An Ensemble of Neural Networks for Online Electron Filtering at the ATLAS Experiment." Journal of Physics: Conference Series. Vol. 1525. No. 1. IOP Publishing, 2020.

[12] Levy, Itamar, and FCAL Collaboration. "Measurement of shower development and its Molière radius with a four-plane LumiCal test setup." Proceedings of the European Physical Society Conference on High Energy Physics. 5-12 July, 2017 Venice, Italy (EPS-HEP2017). Online at http://pos. sissa. it/cgi-bin/reader/conf. cgi? confid= 314, id. 502. 2017.

[13] Seixas, J. M., et al. "Neural second-level trigger system based on calorimetry." Computer Physics Communications 95.2-3 (1996): 143157.

[14] Alberich, L. Cerda. "Photon and electron identification with the ATLAS detector." Proceedings of the 38th International Conference on High Energy Physics (ICHEP2016). 3-10 August 2016. Chicago. 2016.

[15] Kingma, Diederik P., and Jimmy Ba. "Adam: A method for stochastic optimization." arXiv preprint arXiv:1412.6980 (2014).

[16] Ciodaro, T., et al. "Online particle detection with neural networks based on topological calorimetry information." Journal of physics: conference series. Vol. 368. No. 1. IOP Publishing, 2012.

[17] Torres, Rodrigo Coura, et al. "Neural online filtering based on preprocessed calorimeter data." 2009 IEEE Nuclear Science Symposium Conference Record (NSS/MIC). IEEE, 2009.

[18] Simas Filho, Eduardo F., José Manoel de Seixas, and Luiz Pereira Calôba. "Modified post-nonlinear ICA model for online neural discrimination." Neurocomputing 73.16-18 (2010): 2820-2828.

[19] Raschka, Sebastian. "Model evaluation, model selection, and algorithm selection in machine learning." arXiv preprint arXiv:1811.12808 (2018). 